\title{
Rough Approximation Operators in a Topological Ring
}

\author{
Gülay OĞUZ *
}

Siirt University, Faculty of Arts and Sciences, Department of Mathematics, Siirt, Turkey.

Geliş / Received: 08/11/2019, Kabul / Accepted: 20/02/2020

\begin{abstract}
The goal of this article is to examine the concept of topological ring from the perspective of the rough set theory and to introduce the approximation operators in a topological ring with respect to its ideal. Moreover, the definitions of lower topological rough subring and upper topological rough subring are presented. After that, the notion of topological rough ring is described and some related properties are studied.
\end{abstract}

Keywords: Rough set, rough approximations, rough ring, topological rough ring

\section{Bir Topolojik Halkada Kaba Yaklaşım Operatörleri}

$\ddot{\mathbf{O z}}$

$\mathrm{Bu}$ çalışmanın amacı, topolojik halka kavramını kaba küme teorisindeki bakış açısıyla inceleyerek bir topolojik halkada idealine göre alt ve üst yaklaşımları tanıtmaktır. Ayrıca, alt topolojik kaba alt halka ve üst topolojik kaba alt halka tanımları sunulmuştur. Sonrasında, topolojik kaba halka kavramı tanımlanarak ilgili bazı özellikler incelenmiştir.

Anahtar Kelimeler: Kaba küme, kaba yaklaşımlar, kaba halka, topolojik kaba halka.

\section{Introduction}

The classical logic that begins with Aristotle is based on complete and precise information. Over time, the traditional methods for solving many complexproblems that push the boundaries of classical logic in areas such as data analysis,engineering, computer science and medicine have remained insufficient. At this point, theories such as fuzzy sets, rough sets and soft sets proposed as a result of studies on modeling incomplete and uncertain information (Zadeh, 1965; Pawlak, 1982; Molodtsov, 1999).
Rough set theory presented to the world of science by Pawlak (1982) is a powerful mathematical approach for deal with uncertainties. In particular, this theory has managed to attract attention in almost all areas of mathematics by creating a high potential for algebraic, topological and geometrical studies (Kuroki and Mordeson, 1997; Wiweger, 1998; Miao, et al., 2005; Oguz, et al., 2018). After pawlak, initial algebraic studies on rough sets were made by (Iwinski, 1987). Biswas and Nanda (1994) defined the concepts of rough group and rough subgroup. Davvaz (2004) investigated roughness in rings. 
For other algebraic studies on rough sets, see (Davvaz and Mahdavipour, 2006; Kuroki, 1997).

Topology, which is a crucial branch of mathematics, has a wide range of use in almost all branches of mathematics as well as in many real-life applications. In this perspective, it is inevitable to introduce the topological rough concepts by examining the intersection of topology with rough approach. The notion of topological rough spaces was defined by (Wu, et al., 2008). At the same time, they established some important characterization on topological rough spaces. Also, topological rough groups and rough approximations in a topological group were studied by (Bagirmaz, et al., 2016). As an advanced level of this work, Oguz et al (2018) described the notions of lie rough groups and lie rough subgroups. Apart from these studies, many topological studies related to rough set theory have been conducted (Wiweger, 1989; Lashin, et al., 2005; Li, et al., 2012).

On the other hand, many concepts of mathematics have both topological and algebraic characteristics and the concept of topological ring is one of them. In the present work, the topological rings have been studied with rough approximation operators and the notion of topological rough rings has been introduced. Inaddition, the axiomatic features provided for the lower and upper approximation operators of topological rings are constructed. The notions of lower topological rough subring and upper topological rough subring are described. In short, this article is regarded as an important bridge that establishes the relationship between the topological rings and rough set theory. It also contributes to significantly expand the boundaries of topological research on rough sets.

\section{Preliminaries}

This section recalls several main definitions and features of rough sets and rough rings to be used throughout the article. For more details, see to (Pawlak, 1982; Kuroki and Mordeson, 1997; Davvaz, 2004).

Assume that $E$ is a certain set called the universe. Pawlak defined the notion of a rough set as follows:

Definition 2.1 (Pawlak, 1982) A pair (E, $\beta$ ) is called an approximation space, where $\beta$ is a an equivalence relation on the universe $E$.

Definition 2.2 (Pawlak, 1982) Let $M$ be a certain subset of $E$. The lower approximation of $M$ is the union of all the equivalence classes which are completely included in $M$. We denote it as $\underline{\beta}(M)$ such that

$$
\underline{\beta}(M)=\bigcup_{\varepsilon \in \mathrm{E}}\{\beta(\varepsilon): \beta(\varepsilon) \subseteq M\}
$$

Definition 2.3 (Pawlak, 1982) Let $M$ be a certain subset of $E$. The upper approximation of $M$ is the union of all the equivalence classes which have non-empty intersection with the set. We write it as $\bar{\beta}(M)$ such that

$$
\bar{\beta}(M)=\bigcup_{\varepsilon \in E}\{\beta(\varepsilon): \beta(\varepsilon) \cap M \neq \varnothing\}
$$

Definition 2.4 (Pawlak, 1982) Let $M$ be a certain subset of $\mathrm{E}$. The boundary region of $M$ denoted by $\mathrm{S}_{\beta}(M)$ is the set $\mathrm{S}_{\beta}(M)=$ $\bar{\beta}(M)-\underline{\beta}(M)$ in the universe $E$. 
Definition 2.5 (Pawlak, 1982) The subset $A$ is said to be a rough set if the boundary region is different from the empty set, otherwise it is said to be a crisp (exact) set. Furthermore, it is denoted as $\beta(M)=$ $(\beta(M), \bar{\beta}(M))$ if $A$ is a rough set in the approximation space $(\mathrm{E}, \beta)$.

Corollary 2.6 (Pawlak, 1982) Let $M$ and B be two subsets of $E$ in the approximation space (E, $\beta$ ). Then, the following axioms about the lower and upper approximation operators hold:

1. $\underline{\beta}(M) \subseteq X \subseteq \bar{\beta}(M)$,

2. $\underline{\beta}(\varnothing)=\bar{\beta}(\varnothing)=\emptyset, \underline{\beta}(E)=\bar{\beta}(E)=E$,

3. $\underline{\beta}(M \cap N)=\underline{\beta}(M) \cap \underline{\beta}(N)$,

4. $\underline{\beta}(M \cup N) \subseteq \underline{\beta}(M) \cup \underline{\beta}(N)$,

5. $\bar{\beta}(M \cup N)=\bar{\beta}(M) \cup \bar{\beta}(N)$,

6. $\bar{\beta}(M) \cap \bar{\beta}(N)=\bar{\beta}(M \cap N)$,

7. $M \subseteq N \Rightarrow \beta(M) \subseteq \beta(N)$

8. $M \subseteq N \Rightarrow \bar{\beta}(M) \subseteq \bar{\beta}(N)$,

9. $\underline{\boldsymbol{\beta}}(\underline{\boldsymbol{\beta}}(\boldsymbol{M}))=\overline{\boldsymbol{\beta}} \underline{\beta}(\boldsymbol{M})=\underline{\boldsymbol{\beta}}(\boldsymbol{M})$,

10. $\overline{\boldsymbol{\beta}}(\overline{\boldsymbol{\beta}}(M))=\boldsymbol{\beta} \overline{\boldsymbol{\beta}}(M)=\overline{\boldsymbol{\beta}}(M)$.

Here, we will recall the concepts of rough ideal and rough ring to ensure the integrity of this study. Assume that $\mathcal{H}$ is a ring and $\mathcal{K}$ is an ideal of $\mathcal{H}$.

Definition 2.7 (Davvaz, 2004) Let $M$ be a non-empty subset of $\mathcal{H}$. The upper approximation of $\mathrm{M}$ with regard to $\mathcal{K}$ is defined as the set

$$
\overline{\mathcal{K}}(\mathrm{M})=\{\varepsilon \in \mathcal{H}:(\varepsilon+\mathcal{K}) \cap \mathrm{M} \neq \emptyset\}
$$

In this manner, the lower approximation of $\mathrm{M}$ with regard to $\mathcal{K}$ is defined as the set

$$
\underline{\mathcal{K}}(\mathrm{M})=\{\varepsilon \in \mathcal{H}: \varepsilon+\mathcal{K} \subseteq \mathrm{M}\}
$$

Remark 2.8 It is important to note that the relation $\varepsilon \equiv \delta(\bmod \mathcal{K})$ is an equivalence relation, defined as $\varepsilon \equiv \delta(\bmod \mathcal{K})$ if $\varepsilon-\delta \in$ $\mathcal{K}$ for $\varepsilon, \delta \in \mathcal{H}$. In this case, one can say easily that the pair $(\mathcal{H}, \mathcal{K})$ is a approximation space.

Example 2.9 (Davvaz, 2004) Let $\mathcal{H}=\mathbb{Z}_{15}$, $\mathcal{K}=\{0,5,10\} \quad$ and $\quad M=\{1,6,7,8,10,11\}$. Then, we obtain

$$
\overline{\mathcal{K}}(\mathrm{M})=\{0,1,2,3,5,6,7,8,10,11,12,13\}
$$

and

$$
\underline{\mathcal{K}}(\mathbf{M})=\{1,6,11\}
$$

Proposition 2.10 (Davvaz, 2004) Let $\mathcal{P}$ and $\mathcal{K}$ be two ideals of $\mathcal{H}$. Then, $\overline{\mathcal{P}}(\mathcal{K})$ and $\underline{\mathcal{P}}(\mathcal{K})$ are also the ideals of $\mathcal{H}$.

Definition 2.11 (Davvaz, 2004) Let $\mathcal{K}(\mathrm{M})=$ $(\underline{\mathcal{K}}(\mathrm{M}), \overline{\mathcal{K}}(\mathrm{M}))$ be a rough set in the approximation space $(\mathcal{H}, \mathcal{K})$. Then, $\mathcal{K}(\mathrm{M})$ is said to be a rough ideal (resp. subring) if $\underline{\mathcal{K}}(\mathrm{M})$ and $\overline{\mathcal{K}}(\mathrm{M})$ are ideals (resp. subring) of $\mathcal{H}$. Besides, a rough subring is called a rough ring.

Proposition 2.12 (Davvaz, 2004) Let $\mathcal{K}$ be an ideal and $\mathrm{M}$ is a subring of $\mathcal{H}$. Then, $\mathcal{K}(\mathrm{M})$ is a rough ring.

Example 2.13 (Davvaz, 2004) Let us consider $\mathcal{H}=\mathbb{Z}_{15}, \mathcal{K}=\{0,5,10\}$ and $\mathrm{M}=$ $\{0,3,6,9,12\}$. From above proposition, it is easy to see that $\mathcal{K}$ is a ideal and $\mathrm{M}$ is a subring of $\mathcal{H}$ such that $\mathcal{K}(\mathrm{M})=$ $(\underline{\mathcal{K}}(\mathrm{M}), \overline{\mathcal{K}}(\mathrm{M}))$ is a rough ring.

Proposition 2.14 (Davvaz, 2004) Let $\mathcal{P}$ and $\mathcal{K}$ be two ideals of $\mathcal{H}$ and $\mathrm{M}$ is a subring of $\mathcal{H}$. Then, the following conditions are satisfied:

i. $\overline{\mathcal{P}}(M)+\overline{\mathcal{K}}(M)=(\overline{\mathcal{P}+\mathcal{K}})(M)$ 


\section{ii. $\underline{\mathcal{P}}(M)+\underline{\mathcal{K}}(M)=(\underline{\mathcal{P}+\mathcal{K}})(M)$}

\section{Topological Rough Rings}

In this section, we propose the concept of lower and upper approximations in a topological ring with respect to its ideal. Also, we give the definitions of lower topological rough subring and upper topological rough subring, and establish some of their characterizations.

In what follows, $\mathcal{H}$ is a topological ring unless stated otherwise.

Definition 3.1 Let $\mathcal{P}$ be an ideal of the topological ring $\mathcal{H}$. A pair $(\mathcal{H}, \mathcal{P})$ where $\boldsymbol{\varepsilon} \equiv \boldsymbol{\delta}(\bmod \mathcal{P})$ is an equivalence relation on $\mathcal{H}$ is said to be a topological approximation space.

Definition 3.2 Let $(\mathcal{H}, \mathcal{P})$ be a topological approximation space and $\mathbf{M}$ be a nonempty subset of $\mathcal{H}$. Then, the sets

$$
\overline{\mathcal{P}}(\mathrm{M})=\{\varepsilon \in \mathcal{H}:(\varepsilon+\mathcal{P}) \cap \mathrm{M} \neq \emptyset\}
$$

and

$$
\underline{\mathcal{P}}(\mathrm{M})=\{\varepsilon \in \mathcal{H}: \varepsilon+\mathcal{P} \subseteq \mathrm{M}\}
$$

is said to be the lower and upper approximation of the set $\mathrm{M}$ with respect to the ideal $\mathcal{P}$, respectively.

It can be easily shown that these operators provide the following properties:

Proposition 3.3 For all subsets $M, N \subseteq \mathcal{H}$ in the topological approximation space $(\mathcal{H}, \mathcal{P})$

1. $\underline{\mathcal{P}}(\mathrm{M}) \subseteq \mathrm{M} \subseteq \overline{\mathcal{P}}(\mathrm{M})$,

2. $\underline{\mathcal{P}}(\varnothing)=\overline{\mathcal{P}}(\varnothing)=\emptyset, \underline{\mathcal{P}}(\mathcal{H})=\overline{\mathcal{P}}(\mathcal{H})=\mathcal{H}$,

3. $\underline{\mathcal{P}}(\mathrm{M} \cap \mathrm{N})=\underline{\mathcal{P}}(\mathrm{M}) \cap \underline{\mathcal{P}}(\mathrm{N})$,

4. $\underline{\mathcal{P}}(\mathrm{M} \cup \mathrm{N}) \subseteq \underline{\mathcal{P}}(\mathrm{M}) \cup \underline{\mathcal{P}}(\mathrm{N})$,
5. $\overline{\mathcal{P}}(\mathrm{M} \cup \mathrm{N})=\overline{\mathcal{P}}(\mathrm{M}) \cup \overline{\mathcal{P}}(\mathrm{N})$,

6. $\overline{\mathcal{P}}(\mathrm{M}) \cap \overline{\mathcal{P}}(\mathrm{N})=\overline{\mathcal{P}}(\mathrm{M} \cap \mathrm{N})$,

7. $\mathrm{M} \subseteq \mathrm{N} \Rightarrow \underline{\mathcal{P}}(\mathrm{M}) \subseteq \underline{\mathcal{P}}(\mathrm{N}), \overline{\mathcal{P}}(\mathrm{M}) \subseteq \overline{\mathcal{P}}(\mathrm{N})$,

8. $\underline{\mathcal{P}}(\underline{\mathcal{P}}(\mathrm{M}))=\overline{\mathcal{P}}(\underline{\mathcal{P}}(\mathrm{M}))=\underline{\mathcal{P}}(\mathrm{M})$,

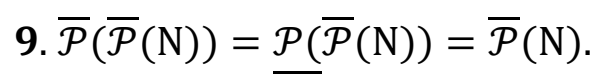

10. $\overline{\mathcal{P}}(\mathcal{P}+\varepsilon)=\underline{\mathcal{P}}(\mathcal{P}+\varepsilon)$ for all $\varepsilon \in \mathcal{H}$.

Example 3.4 Consider the topological ring $\mathcal{H}=\mathbb{Z}_{6}$ with the the discrete topology. For $\mathcal{H}$ with the ideal $\mathcal{P}=\{0,3\}$, it is clear that if $M=\{0,1,3,5\}$ then $\overline{\mathcal{P}}(M)=\{0,1,2,3,4,5\}$ and $\underline{\mathcal{P}}(\mathrm{M})=\{0,3\}$.

Proposition 3.5 Let $\mathcal{P}, \mathcal{K}$ be two ideals of the topological ring $\mathcal{H}$ and $\mathcal{P} \subseteq \mathcal{K}$. Then, for $\mathrm{M} \subseteq \mathcal{H}$

i. $\overline{\mathcal{P}}(\mathrm{M}) \subseteq \overline{\mathcal{K}}(\mathrm{M})$,

ii. $\underline{\mathcal{K}}(\mathrm{M}) \subseteq \underline{\mathcal{P}}(\mathrm{M})$.

Proof. i. Assume $\varepsilon \in \overline{\mathcal{P}}(\mathrm{M})$. Then $(\varepsilon+$ $\mathcal{P}) \cap \mathrm{M} \neq \emptyset$. Since $\mathcal{P} \subseteq \mathcal{K},(\varepsilon+\mathcal{K}) \cap \mathrm{M} \neq$ $\emptyset$. This implies $\varepsilon \in \overline{\mathcal{K}}(\mathrm{M})$. Hence $\overline{\mathcal{P}}(\mathrm{M}) \subseteq$ $\overline{\mathcal{K}}(\mathrm{M})$.

Similarly, we can prove the second condition as follows:

ii. Let $\varepsilon \in \underline{\mathcal{K}}(M)$. Then $(\varepsilon+\mathcal{K}) \subseteq M$. Since $\mathcal{P} \subseteq \mathcal{K}$, so $(\varepsilon+\mathcal{P}) \subseteq M$. Thus $\varepsilon \in \underline{\mathcal{K}}(M)$. This shows that $\underline{\mathcal{K}}(M) \subseteq \underline{\mathcal{P}}(M)$.

After this proposition, we can easily obtain the following corollary.

Corollary 3.6 Let $\mathcal{P}, \mathcal{K}$ be two ideals of the topological ring $\mathcal{H}$. Then, for $\mathrm{M} \subseteq \mathcal{H}$

i. $(\overline{\mathcal{P} \cap \mathcal{K}})(\mathrm{M}) \subseteq \overline{\mathcal{P}}(\mathrm{M}) \cap \overline{\mathcal{K}}(\mathrm{M})$,

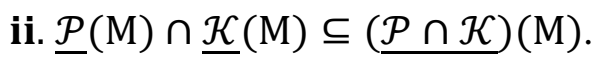


Proof. i. Suppose $\varepsilon \in(\overline{\mathcal{P} \cap \mathcal{K}})(M)$. Then $(\varepsilon+(\mathcal{P} \cap \mathcal{K})) \cap M \neq \emptyset$, that is mean

$$
(\varepsilon+\mathcal{P}) \cap M \neq \emptyset \text { and }(\varepsilon+\mathcal{K}) \cap M \neq \varnothing
$$

implies $\varepsilon \in \overline{\mathcal{P}}(M)$ and $\varepsilon \in \overline{\mathcal{K}}(M)$. Therefore, we have $(\overline{\mathcal{P} \cap \mathcal{K}})(M) \subseteq \overline{\mathcal{P}}(M) \cap \overline{\mathcal{K}}(M)$.

ii. Let $\varepsilon \in \underline{\mathcal{P}}(M) \cap \underline{\mathcal{K}}(M)$. Then we have $\varepsilon \in$ $\underline{\mathcal{P}}(M)$ and $\varepsilon \in \mathcal{P}(M)$, that is $(\varepsilon+\mathcal{P}) \subseteq M$ and $(\varepsilon+\mathcal{K}) \subseteq M$. Thus $(\varepsilon+(\mathcal{P} \cap \mathcal{K})) \subseteq$ $M$, so that $\varepsilon \in(\underline{\mathcal{P} \cap \mathcal{K}})$, which proves the proposition.

Proposition 3.7 If $\mathcal{P}$ and $\mathcal{K}$ are two ideals of the topological ring $\mathcal{H}$, then $\overline{\mathcal{P}}(\mathcal{K})$ and $\underline{\mathcal{P}}(\mathcal{K})$ are two ideals of the topological ring $\mathcal{H}$.

Proof. Suppose that $\mathcal{P}$ and $\mathcal{K}$ are two ideals of the topological ring $\mathcal{H}$. For all $\varepsilon, \delta \in$ $\overline{\mathcal{P}}(\mathcal{K})$ and $\lambda \in \mathcal{H}, \quad(\varepsilon+\mathcal{P}) \cap \mathcal{K} \neq \varnothing$ and $(\delta+\mathcal{P}) \cap \mathcal{K} \neq \emptyset$ such that $(\varepsilon-\delta+\mathcal{P}) \cap$ $\mathcal{K} \neq \varnothing \quad$ and $\quad(\lambda \varepsilon+\mathcal{P}) \cap \mathcal{K} \neq \varnothing \quad$ imply $\varepsilon-\delta \in \overline{\mathcal{P}}(\mathcal{K})$ and $\lambda \varepsilon \in \overline{\mathcal{P}}(\mathcal{K})$. Hence, $\overline{\mathcal{P}}(\mathcal{K})$ is an ideal of the topological ring $\mathcal{H}$.

Likewise, we can prove that $\underline{\mathcal{P}}(\mathcal{K})$ is also an ideal of the topological ring $\mathcal{H}$.

In the sequel, it is easy to verify that

Proposition 3.8 If $\mathcal{P}$ is a ideal of the topological ring $\mathcal{H}$ and $\mathrm{T}$ is a subring of $\mathcal{H}$ , then $\overline{\mathcal{P}}(\mathrm{T})$ and $\underline{\mathcal{P}}(\mathrm{T})$ are two subrings of the topological ring $\mathcal{H}$.

Proof. Let $\varepsilon, \delta \in \overline{\mathcal{P}}(\mathrm{T})$ such that $(\varepsilon+\mathcal{P}) \cap$ $\mathrm{G} \neq \varnothing$ and $(\delta+\mathcal{P}) \cap \mathrm{T} \neq \varnothing$. Then, there exist an element $a \in(\varepsilon+\mathcal{P}) \cap \mathrm{T}$ and $\mathrm{b} \in(\delta+\mathcal{P}) \cap \mathrm{T}$. Since $\mathrm{T}$ is a subring of $\mathcal{H}$, $\mathrm{a}-\mathrm{b} \in \mathrm{T}, \mathrm{ab} \in \mathrm{T}$, and hence

$$
\begin{gathered}
\mathrm{a}-\mathrm{b} \in(\varepsilon+\mathcal{P})-(\delta+\mathcal{P})=\varepsilon-\delta+\mathcal{P}, \\
\mathrm{ab} \in(\varepsilon+\mathcal{P})(\delta+\mathcal{P})=\varepsilon \delta+\mathcal{P}
\end{gathered}
$$

which gives
$(\varepsilon-\delta+\mathcal{P}) \cap \mathrm{T} \neq \varnothing$ and $(\varepsilon \delta+\mathcal{P}) \cap \mathrm{T} \neq$ $\emptyset$.

This shows that $\overline{\mathcal{P}}(\mathrm{G})$ is a subring of the topological ring $\mathcal{H}$.

Analogously, it can be easily shown that $\underline{\mathcal{P}}(\mathrm{T})$ is a subring of the topological ring $\mathcal{H}$.

Let us now describe the concept of topological rough ring.

Definition 3.9 Let $\mathcal{P}(\mathrm{M})=(\underline{\mathcal{P}}(\mathrm{M}), \overline{\mathcal{P}}(\mathrm{M}))$ be a rough set in the topological approximation space $(\mathcal{H}, \mathcal{P})$. If $\underline{\mathcal{P}}(\mathrm{M})$ is a topological subring of $\mathcal{H}$, then $\mathcal{P}(\mathrm{M})$ is said to be a lower topological rough subring. Conversely, if $\overline{\mathcal{P}}(\mathrm{M})$ is a topological subring of $\mathcal{H}$, then $\mathcal{P}(\mathrm{M})$ is said to be a upper topological rough subring.

Definition 3.10 Let $\mathcal{P}(\mathrm{M})=(\underline{\mathcal{P}}(\mathrm{M}), \overline{\mathcal{P}}(\mathrm{M}))$ be a rough set in the topological approximation space $(\mathcal{H}, \mathcal{P})$. If both $\underline{\mathcal{P}}(\mathrm{M})$ and $\overline{\mathcal{P}}(\mathrm{M})$ are the topological subrings of $\mathcal{H}$, then $\mathcal{P}(\mathrm{M})$ is called a topological rough ring.

Proposition 3.11 If $\mathcal{P}$ is a ideal of the topological ring $\mathcal{H}$ and $\mathrm{T}$ is a topological subring of $\mathcal{H}$, then $\mathcal{P}(\mathrm{T})=(\underline{\mathcal{P}}(\mathrm{T}), \overline{\mathcal{P}}(\mathrm{G}))$ is a topological rough ring.

Proof. Assume that $\mathcal{P}$ is a ideal of the topological ring $\mathcal{H}$ and $\mathrm{T}$ is a topological subring of $\mathcal{H}$. Since by Proposition 3.8, $\underline{\mathcal{P}}(\mathrm{G})$ and $\overline{\mathcal{P}}(\mathrm{G})$ are two topological subrings of $\mathcal{H}$. Therefore, the pair $\mathcal{P}(\mathrm{G})=$ $(\underline{\mathcal{P}}(\mathrm{T}), \overline{\mathcal{P}}(\mathrm{U}))$ is a topological rough ring.

Proposition 3.12 Let $\mathcal{P}$ and $\mathcal{K}$ be two ideals of the topological ring $\mathcal{H}$ and let $\mathrm{T}$ be a topological subring of $\mathcal{H}$. Then

i. $\overline{\mathcal{P}}(\mathrm{T})+\overline{\mathcal{K}}(\mathrm{T})=(\overline{\mathcal{P}+\mathcal{K}})(\mathrm{T})$,

ii. $\underline{\mathcal{P}}(\mathrm{G})+\underline{\mathcal{K}}(\mathrm{T})=(\underline{\mathcal{P}+\mathcal{K}})(\mathrm{T})$. 
Proof. i) For the ideals $\mathcal{P}, \mathcal{K}$ of the topological ring $\mathcal{H}$, it can be written that $\mathcal{P} \subseteq \mathcal{P}+\mathcal{K} \quad$ and $\quad \mathcal{K} \subseteq \mathcal{P}+\mathcal{K}$. Also by Proposition 3.5 , we have $\overline{\mathcal{P}}(\mathrm{G}) \subseteq$ $(\overline{\mathcal{P}+\mathcal{K}})(\mathrm{G})$ and $\overline{\mathcal{K}}(\mathrm{T}) \subseteq(\overline{\mathcal{P}+\mathcal{K}})(\mathrm{T})$ for the topological subring $\mathcal{V}$ of $\mathcal{H}$. This implies that

$\overline{\mathcal{P}}(\mathrm{T})+\overline{\mathcal{K}}(\mathrm{T}) \subseteq(\overline{\mathcal{P}+\mathcal{K})}(\mathrm{T})$.

Conversely, suppose that $\varepsilon \in(\overline{\mathcal{P}+\mathcal{K}})(\mathrm{T})$. Then, $(\varepsilon+(\mathcal{P}+\mathcal{K})) \cap \mathrm{T} \neq \varnothing$ that means there is an element $\delta$ of $\mathcal{P}$ such that $(\varepsilon+\delta+$ $\mathcal{K}) \cap \mathrm{T} \neq \emptyset$, which implies $\varepsilon+\delta \in \overline{\mathcal{K}}(\mathrm{T})$. Conversely, as $-\delta \in \mathcal{P}$ so $(-\delta+\mathcal{P}) \cap \mathrm{T}=$ $\mathcal{P} \cap \mathcal{Q} \neq \emptyset$. Thus $-\delta \in \overline{\mathcal{P}}(\mathrm{T})$. This shows that

$$
\varepsilon=-\delta+(\varepsilon+\delta) \in \overline{\mathcal{P}}(\mathrm{G})+\overline{\mathcal{K}}(\mathrm{T})
$$

, hence $(\overline{\mathcal{P}+\mathcal{K})}(\mathrm{G}) \subseteq \overline{\mathcal{P}}(\mathrm{G})+\overline{\mathcal{K}}(\mathrm{T})$.

It can also be easily proved that the condition ii is satisfied.

Note that let $\mathcal{H}$ and $\mathcal{H}^{\prime}$ be two topological rings. It is well known from ring theory that if $\psi: \mathcal{H} \rightarrow \mathcal{H}^{\prime}$ is a topological ring homomorphism, the kernel $\Omega$ of $\psi$ is an ideal of $\mathcal{H}$.

Proposition 3.13 Let $\psi: \mathcal{H} \rightarrow \mathcal{H}^{\prime}$ be a topological ring homomorphism and $\mathrm{M} \subseteq \mathcal{H}$. Then, we have $\psi(\bar{\Omega}(\mathrm{M}))(\bar{\Omega}(\mathrm{M}))=\psi(\mathrm{M})$.

Proof. By noting $\mathrm{M} \subseteq \bar{\Omega}(\mathrm{M})$, it is easy to observe that $\psi(\mathrm{M}) \subseteq \psi(\bar{\Omega}(\mathrm{M}))$.

On the other hand, take an element $\delta \in$ $\psi(\bar{\Omega}(\mathrm{M}))$. By the assumption, there exists an element $\varepsilon \in \bar{\Omega}(\mathrm{M})$ such that $\psi(\varepsilon)=\delta$ holds. Hence, we have $(\varepsilon+\Omega) \cap \mathrm{M} \neq \varnothing$. Note that there exists an element $\mathrm{a} \in(\varepsilon+\Omega) \cap \mathrm{M}$, so that $\mathrm{a}=\varepsilon+\mathrm{b}$ holds for some $\mathrm{b} \in \Omega$, in other words $\varepsilon=\mathrm{a}-\mathrm{b}$. Then, it holds that

$$
\begin{gathered}
\delta=\psi(\varepsilon)=\psi(a-b)=\psi(a)-\psi(b) \\
\in \Psi(M)
\end{gathered}
$$

and that $\psi(\bar{\Omega}(M)) \subseteq \psi(M)$ which completes the proof.

Example 3.14 Consider the topological rings $\mathbb{Z}_{12}$ and $\mathbb{Z}_{4}$ with the discrete topology. The topological ring homomorphism

$\psi: \mathbb{Z}_{12} \rightarrow \mathbb{Z}_{4}$ is defined by $\psi(1)=3$

.Then, $\Omega=\{0,4,8\}$ is the kernel of $\psi$. Choose $M=\{1,3,7,8\}$. Here it is easy to verify that $\bar{\Omega}(\mathrm{M})=\{0,1,3,4,5,7,8,9,11\}$ so that $\psi(\bar{\Omega}(\mathrm{M}))=3=\psi(\mathrm{M})$.

We introduce another concept and conclude this section with two results around the topological equivalent form of rough approximations here:

Definition 3.15 Let $\mathcal{P}$ be a ideal of the topological ring $\mathcal{H}$ and $\mathrm{M} \subseteq \mathcal{H}$. The equivalent forms of rough approximations are defined as

$$
\overline{\mathcal{P}(\mathrm{M})}=\{\varepsilon \in \mathcal{H} / \mathcal{P}:(\varepsilon+\mathcal{P}) \cap \mathrm{M} \neq \emptyset\}
$$

and

$$
\underline{\mathcal{P}(\mathrm{M})}=\{\varepsilon \in \mathcal{H} / \mathcal{P}: \varepsilon+\mathcal{P} \subseteq \mathrm{M}\}
$$

Proposition 3.16 Let $\mathcal{P}$ and $\mathcal{K}$ be two ideal of the topological ring $\mathcal{H}$. Then, $\overline{\mathcal{P}(\mathcal{K})}$ and $\mathcal{P}(\mathcal{K})$ are also two ideal of $\mathcal{H} / \mathcal{P}$.

Proof. Suppose $\varepsilon+\mathcal{P}, \delta+\mathcal{P} \in \overline{\mathcal{P}(\mathcal{K})}$ and $\mathrm{z}+\mathcal{P} \in \mathcal{H} / \mathcal{P}$. Then $(\varepsilon+\mathcal{P}) \cap \mathcal{K} \neq \emptyset$ and $(\delta+\mathcal{P}) \cap \mathcal{K} \neq \varnothing$, which show that there exists $\mathrm{a} \in(\varepsilon+\mathcal{P}) \cap \mathcal{K}$ and $\mathrm{b} \in(\delta+\mathcal{P}) \cap$ $\mathcal{K}$. By assumption, $\mathrm{a}-\mathrm{b} \in \mathcal{K}$ and za $\in \mathcal{K}$ since $\mathcal{K}$ is an ideal of the topological ring $\mathcal{H}$. Clearly,

$\mathrm{a}-\mathrm{b} \in(\varepsilon+\mathcal{P})-(\delta+\mathcal{P})=(\varepsilon-\delta)+\mathcal{P}$ 
and

$$
\mathrm{za} \in \mathrm{z}(\varepsilon+\mathcal{P})=\mathrm{z} \varepsilon+\mathcal{P}
$$

. Hence $(\varepsilon-\delta+\mathcal{P}) \cap \mathcal{K} \neq \emptyset, \quad(\mathrm{z} \varepsilon+\mathcal{P}) \cap$ $\mathcal{K} \neq \emptyset$. Therefore, $(\mathrm{z}+\mathcal{P})(\varepsilon+\mathcal{P}) \in \overline{\mathcal{P}(\mathcal{K})}$ and $(\varepsilon+\mathcal{P})-(\delta+\mathcal{P}) \in \overline{\mathcal{P}(\mathcal{K})}$, so that $\overline{\mathcal{P}(\mathcal{K})}$ is an ideal of the topological ring $\mathcal{H} / \mathcal{P}$.

Conversely, let $\varepsilon+\mathcal{P}, \delta+\mathcal{P} \in \mathcal{P}(\mathcal{K})$ and $\mathrm{z}+\mathcal{P} \in \mathcal{H} / \mathcal{P}$ such that $(\varepsilon+\mathcal{P}) \subseteq \mathcal{K}$ and $(\delta+\mathcal{P}) \subseteq \mathcal{K}$. Since $\mathcal{K}$ is an ideal of the topological ring $\mathcal{H}$, then we have

$$
(\varepsilon+\mathcal{P})-(\delta+\mathcal{P}) \subseteq \mathcal{K}=(\varepsilon-\delta)+\mathcal{P}
$$

and

$$
\mathrm{z}(\varepsilon+\mathcal{P})=\mathrm{z} \varepsilon+\mathcal{P} \subseteq \mathcal{K}
$$

Thus, $(\varepsilon+\mathcal{P})-(\delta+\mathcal{P}) \in \mathcal{P}(\mathcal{K})$ and $(\mathrm{z}+$ $\mathcal{P})(\varepsilon+\mathcal{P}) \in \underline{\mathcal{P}(\mathcal{K})}$, and we say that $\underline{\mathcal{P}(\mathcal{K})}$ is an ideal of the topological ring $\mathcal{H} / \mathcal{P}$.

Likewise, it is straighforward to see that

Corollary 3.17 Let $\mathcal{P}$ be an ideal of the topological ring $\mathcal{H}$ and $\mathrm{T}$ be a topological subring of $\mathcal{H}$. Then, $\overline{\mathcal{P}(\mathrm{T})}$ and $\mathcal{P}(\mathrm{T})$ are also two topological subrings of $\mathcal{H} / \mathcal{P}$.

\section{References}

Pawlak, Z. 1982. Rough sets. Int. J. Comput. Inform. Sci., 11: 341-356.

Zadeh, L. A. 1965. Fuzzy sets. Inform. Control. 8: 338-353.

Molodtsov, D. A.1999. Soft set theory-First results. Comput. Math. Appl. 37:19-31.

Wiweger, R. 1989. On Topological Rough Sets. Bull. Pol. Ac. Math.37: 89-93.
Biswas, R, Nanda S.1994. Rough groups and rough subgroups. Bull. Pol. Acad. Sci. Math. 42: 251-254.

Bagirmaz, N, Icen, I, Ozcan, A.F. 2016.Topological Rough Groups. Topol. Algebra Appl. .4: 31-38.

Kuroki, N, Mordeson, J.N.1997. Structure of rough sets and rough groups. J. Fuzzy Math. 5:183-191.

Davvaz, B. 2004. Roughness in rings. Inform. Sci; 164: 147-163.

Kuroki, N.1997. Rough ideals in semigroups. Inform. Sci; 100:139-163.

Davvaz, B, Mahdavipour, M. 2006. Roughness in modules. Inform. Sci; 176: 3658-3674.

Wiweger, A. 1998. On topological rough sets. Bull. Polish Acad. Sci. Math; 37: 5162.

Lashin, E.F, Kozae, A. M, Abo Khadra, A.A, Medhat, T. 2005. Rough set theory for topological spaces. Int. J. Aprrox. Reason; 40: 35-43.

Li, Z, Xie, T, Li, Q. 2012. Topological structure of generalized rough sets. Comput. Math. with Appl; 63: 1066-1071.

Oguz, G, Icen I, Gursoy, M.H. 2018. Lie Rough Groups. Filomat; 32: 5735-5741.

Miao, D, Han, S, Li, D, Sun, L. 2005. Rough Group, Rough Subgroup and Their Properties. LNCS; 3641: 104-113.

Iwinski J.1987. Algebraic approach to rough sets. Bull Polish Acad Sci Math; 35 : 673683. 
Wu, Q, Wang, T, Huang, Y, Li, J. 2008.

Topology Theory on Rough Sets. IEEE

Transactions on Systems, Man and

Cybernetics;38: 68-77. 